\title{
The International Liquid Mirror Telescope (ILMT) as a Variability Time Machine
}

\author{
Joël Poels ${ }^{1}$, Ermanno Borra ${ }^{2}$, Paul Hickson ${ }^{3}$, Ram Sagar $^{4}$, \\ Przemyslaw Bartczak ${ }^{5}$, Ludovic Delchambre ${ }^{1}$, François Finet $^{1}$, \\ Serge Habraken ${ }^{1}$, Jean-Pierre Swings ${ }^{1}$ and Jean Surdej ${ }^{1}$ \\ ${ }^{1}$ AEOS, University of Liège, B-4000, Sart Tilman, Belgium \\ email: poels@astro.ulg.ac.be \\ ${ }^{2}$ Dept. of Physics, Laval University, Canada \\ ${ }^{3}$ Dept of Physics and Astronomy, UBC, Canada \\ ${ }^{4}$ Dept of Astronomy, ARIES, Nainital, India \\ ${ }^{5}$ Dept of Astronomy of A. Mickiewicz University, Poznan, Poland
}

\begin{abstract}
During the year 2012 the International Liquid Mirror Telescope (a collaboration between astronomical institutions in Belgium, Canada, India and Poland) wil see first light. The instrument will provide substantial, in-depth sky coverage and make an unprecedented number of nightly observations.
\end{abstract}

\section{Current Status of the ILMT Project}

The science achievable with this unique instrument is exciting in terms of variability studies, and includes possible cosmological inference. Details of the ILMT in particular can be found on our Web pages, whose URLs are given below. The Website offers access to many public documents featuring pioneer papers dealing with Liquid-Mirror technology, as well as images and videos which we are proud to share. One will also find there some more didactic documents for those interested in this upcoming and promising technology.

Large sections of the ILMT equipment have been shipped to India recently (2011 December), though other parts of the assembly are still in a building phase. Many issues and technical problems were solved during the past two years. Of those, the chief one was related to the quality of the mercury surface. As the objective of the project is to achieve a surface quality close to that of a glass mirror with similar dimensions, we carried out extensive tests and found that if we can avoid surface waves (concentric waves due to vibrations, spiral waves due to rotation, wind, etc.) we achieve a surface accuracy of $\lambda / 2$ when the mercury layer is $1 \mathrm{~mm}$ thick (or even less); see Fig. 1. In order to carry out those measurements and correct such dynamic surface defects we thought that shooting an incident laser beam and capturing its reflection with a dedicated camera could help. We then analysed the signal recorded by the camera using using algorithms based on Fourier transforms to disentangle and characterize the waves. The analysis then guided the tweaking and fine-tuning of a few parameters, and finally we arrived at the expected performance figures. It was also recognised that the mirror container (Fig. 2) is a key component of the system. By meeting all the stated specifications for properties such as rigidity and temperature stability we could be sure that the mirror surface would be as perfect as possible and without distorting wavelets.

The ILMT is erected vertically (Fig. 3), a design which admittedly incorporates both advantages and drawbacks. The latter mainly stem from the fact that objects passing above the ILMT FOV (field of view) do not follow a straight line, and as a consequence 


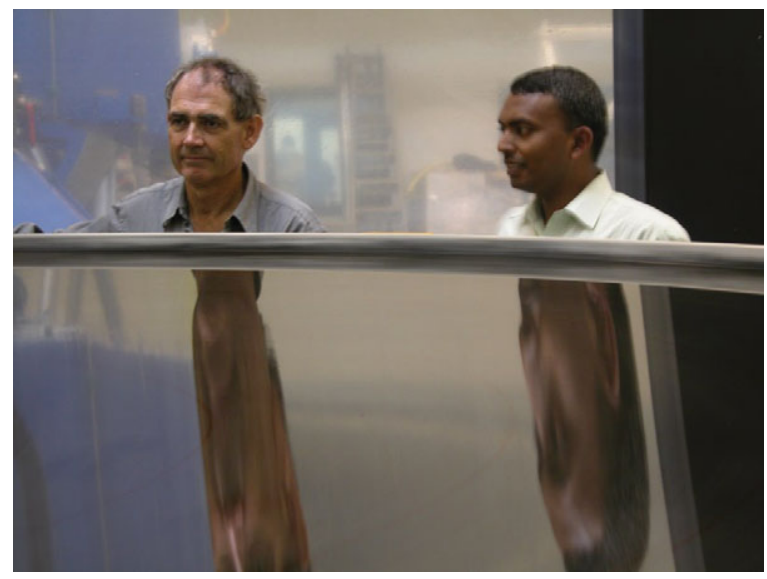

Figure 1. The high quality of the mirror surface.

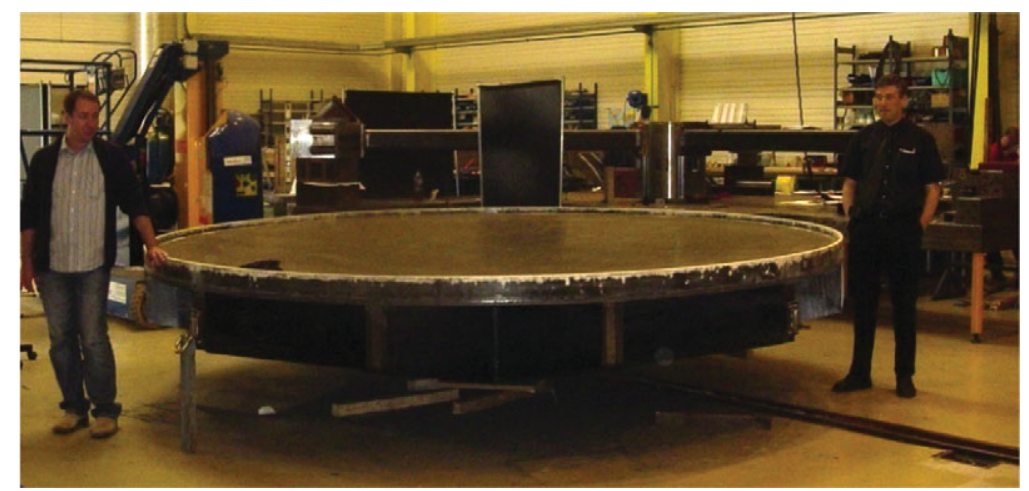

Figure 2. The mirror container is a key component of the system. It must fulfil strict specifications of rigidity and temperature stability in order to guarantee a final shape that is as perfect as possible and to avoid the formation of wavelets on the surface of the mercury.

the image of a point-like source will be degraded and cannot be fitted with a gaussian "seeing" profile. Furthermore, the integration time across the FOV is not the same, and will depend on the declination of the object. In practical terms that effect will depend on the terrestrial latitude where the ILMT is installed. To correct such effects, we designed and built a dedicated corrector that enabled us to eliminate the (well known) Time Delay Integration (TDI) effect. Since the correction is latitude dependent, a dedicated corrector is needed to compensate for the latitude correction of each installation site.

The cost of building such a telescope is roughly 1/50 that of building a conventional instrument of the same class. Even if the ILMT points only to the zenith, that is nevertheless an ideal observing mode since the airmass stays roughly the same, and in addition, pointing to the zenith guarantees the best air transparency. Regarding the choice of the filters, the main one $\left(i^{\prime}\right)$ allows observations for a maximum number of nights because its spectral range is less sensitive to the bright phases of the moon. The camera will be equipped with a set of additional filters $\left(g^{\prime}, r^{\prime}\right.$, shutter), which can be selected according to need. Since the travel time of an object over the FOV is constant, the CCD camera could be dazzled by bright objects; however, such eventualities can obviously be predicted in advance and the shutter activated for as long as it is needed according to the brightness of the source. 


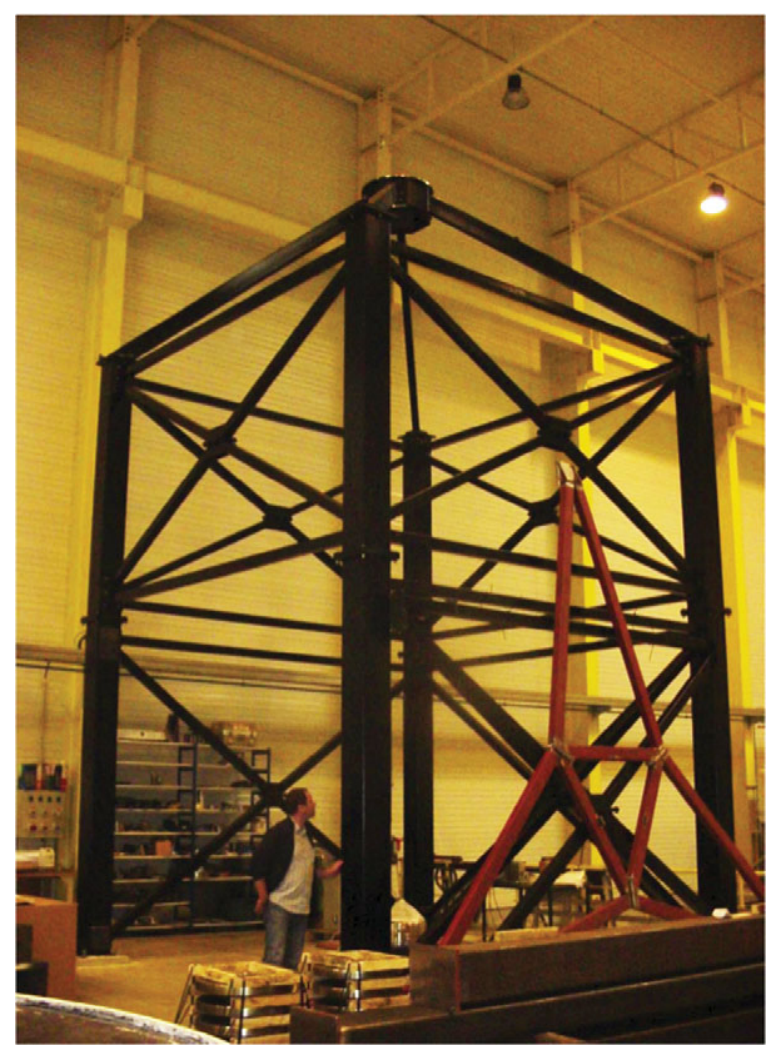

Figure 3. Vertical fixed structure. Focal length $=8 \mathrm{~m}$.

If we were to observe on each night the same $30^{\prime}$-wide strip of sky and apply a systematic image subtraction method to the current and the reference image, there is little doubt that interesting extragalactic objects, as well as other variable ones, will be discovered, and can then be monitored on a daily basis.

We designed an analysis routine so as to preserve data integrity. A strip of night sky was sliced into smaller strips of $4 \mathrm{~K} \times 4 \mathrm{~K}$ pixels, and we applied a software framework which (among other tasks) allows the registration of the location of local/remote rough images into a Relational Database Management System. That database is the heart of a clustering architecture which permits the reduction of multiple images in parallel, using tools such as an Object Oriented $(\mathrm{OO}) \mathrm{C}++$ code that relies on CORBA middleware. Then, when a new ILMT image is stored, various pipelines can be triggered. $\mathrm{C}++$ pipelines can run independently and can achieve many different scientific tasks; some can compute accurately the astrometry and photometry for a detected image feature, while others can provide light curves of objects of interest that are signalled through a Web-browser request which is triggered by users. The potential of this system is vast, within obvious hardware limitations.

Further details of this project can be found on the International Liquid Mirror Telescope Homepage: http://www . aeos.ulg.ac.be/LMT 